\title{
Fabrication and Corrosion Resistance of Superhydrophobic Hydroxide Zinc Carbonate Film on Aluminum Substrates
}

\author{
Jin Liang, ${ }^{1,2}$ Yunchu $\mathrm{Hu}^{2}$ Yiqiang $\mathrm{Wu},{ }^{2}$ and Hong Chen ${ }^{1,2}$ \\ ${ }^{1}$ Institute of Powder Metallurgy, Central South University, Hunan, Changsha 410083, China \\ ${ }^{2}$ Department of Materials Science and Engineering, Central South University of Forestry and Technology, \\ Hunan, Changsha 410004, China \\ Correspondence should be addressed to Jin Liang; ljcfstu@163.com
}

Received 29 December 2012; Accepted 24 January 2013

Academic Editor: Li Li

Copyright (C) 2013 Jin Liang et al. This is an open access article distributed under the Creative Commons Attribution License, which permits unrestricted use, distribution, and reproduction in any medium, provided the original work is properly cited.

\begin{abstract}
Superhydrophobic hydroxide zinc carbonate (HZC) films were fabricated on aluminum substrate through a convenient in situ deposition process. Firstly, HZC films with different morphologies were deposited on aluminum substrates through immersing the aluminum substrates perpendicularly into aqueous solution containing zinc nitrate hexahydrate and urea. Secondly, the films were then modified with fluoroalkylsilane (FAS: $\left.\mathrm{CH}_{3}\left(\mathrm{CF}_{2}\right)_{6}\left(\mathrm{CH}_{2}\right)_{3} \mathrm{Si}\left(\mathrm{OCH}_{3}\right)_{3}\right)$ molecules by immersing in absolute ethanol solution containing FAS. The morphologies, hydrophobicity, chemical compositions, and bonding states of the films were analyzed by scanning electron microscopy (SEM), water contact angle measurement (CA), Fourier transform infrared spectrometer (FTIR), and X-ray photoelectron spectroscopy (XPS), respectively. It was shown by surface morphological observation that HZC films displayed different microstructures such as microporous structure, rose petal-like structure, block-shaped structure, and pinecone-like structure by altering the deposition condition. A highest water contact angle of $156.2^{\circ}$ was obtained after FAS modification. Moreover, the corrosion resistance of the superhydrophobic surface on aluminum substrate was investigated using electrochemical impedance spectroscopy (EIS) measurements. The EIS measurements' results revealed that the superhydrophobic surface considerably improved the corrosion resistance of aluminum.
\end{abstract}

\section{Introduction}

Aluminum and aluminum alloys have been widely used in household and industrial fields such as marine, autoaviation, and aerospace industries, owing to their significant advantages, including high-specific strength, excellent heat and electric conductivities, and low-specific weight $[1,2]$. Also, aluminum has high resistance to corrosion due to the presence of a thin, compact oxide film that spontaneously formed on the metal surface. However, aluminum is active and prone to corrosion in contact with water, especially in corrosive environment such as in aqueous solution containing $\mathrm{Cl}^{-}$ions [3], then it limits the scope of application of aluminum and aluminum alloy. So, it is necessary to enhance the corrosion resistance property of aluminum in corrosive environment which will greatly extend their applications as engineering materials.
In recent years, inspired by the nature, superhydrophobic surfaces with water contact angle of larger than $150^{\circ}$ have attracted much attention due to their self-cleaning and waterrepelling properties [4-6]. Investigation on the representative example of the natural superhydrophobic surface, lotus leaf, revealed that the superhydrophobicity stemmed from the cooperation of wax-like component on leaf surface and surface roughness at micro- and nanoscales [7]. In this way, people developed two strategies to fabricate artificial superhydrophobic surfaces: roughen the surface of hydrophobic materials or chemically modify a rough surface with low surface energy material [8-10]. Traditionally, superhydrophobic metallic surfaces were produced by introducing microstructured compounds modified with low surface energy material onto substrates [11]. Till now, various techniques have been employed to create rough surfaces with different microstructures, such as electrochemical deposition [12], anodization 
[13], phase separation [14], sol-gel process [15], layer-by-layer assembly [16, 17], chemical etching method [9].

Taking advantage of the characteristics of water repellency and low adhesion, superhydrophobic treatments have been applied on various engineering material surfaces such as steel, copper, zinc, titanium, and magnesium to improve their anticorrosion performances by providing an effective barrier to keep water, moisture, and corrosive medium away from contacting and reacting with the metal [18-24]. For instance, Ishizaki et al. $[20,21]$ promoted rapid formation of superhydrophobic surfaces on magnesium alloy by modified microstructured cerium oxide film with fluoroalkylsilane and the anticorrosion resistance of magnesium was significantly improved by the superhydrophobic treatment. Hu et al. [22] fabricated a superhydrophobic $\mathrm{TiO}_{2}$ film with water contact angle greater than $170^{\circ}$ on the Hastelloy substrate after modification with fluoroalkylsilane molecules. Zhang et al. [23] prepared superhydrophobic films on titanium as effective corrosion barriers via the combination of electrochemical oxidation and modification of perfluorooctyltriethoxysilane. Su et al. [24] discussed the tunable wettability of hydroxide zinc carbonate (HZC) film on zinc plates from superhydrophilic to superhydrophobic by altering the type of solvent, but no further study on anticorrosion properties was reported.

In this paper, we attempt to present a facile method for fabricating superhydrophobic surfaces through modulating different morphologies of hydroxide zinc carbonate (HZC) films deposited on aluminum substrate by altering deposition conditions. The transformation of morphologies and wettability of the sample after FAS modification were analyzed. The corrosion resistance property of the superhydrophobic sample was also investigated.

\section{Experimental Section}

2.1. Deposition of HZC Film on Aluminum Substrate. All chemicals were of analytical grade and used without further purification. The HZC films were fabricated through an in situ deposition process; $2 \times 2 \mathrm{~cm}^{2}$ aluminum plates (99.5\%) with a thickness of $1.5 \mathrm{~mm}$ were used as the substrates for the deposition. The substrates were immersed perpendicularly in $0.5 \mathrm{M}$ zinc nitrate aqueous solution containing different molar ratios of urea in a 3-neck, round bottom flask equipped with a Teflon-coated magnetic stir bar and reflux condenser and stirred at $95^{\circ} \mathrm{C}$ for $1.5 \mathrm{~h}$. Then the aluminum substrates were washed several times with deionized water, were blown dry, finally were placed in an oven at $80^{\circ} \mathrm{C}$ for $1 \mathrm{~h}$.

2.2. Modification of the Surfaces. The coated aluminum substrates were immersed in $50 \mathrm{~mL}$ of absolute ethanol solution containing $1 \mathrm{~mL}$ of FAS $\left(\mathrm{CH}_{3}\left(\mathrm{CF}_{2}\right)_{6}\left(\mathrm{CH}_{2}\right)_{3} \mathrm{Si}\left(\mathrm{OCH}_{3}\right)_{3}\right)$ for $24 \mathrm{~h}$ at room temperature. The samples were then thoroughly washed with absolute ethanol several times, were blown dry, finally were placed in an oven at $60^{\circ} \mathrm{C}$ for $1 \mathrm{~h}$.

2.3. Characterization. The surface morphologies of the obtained samples were investigated using a scanning electron microscopy (NOVATM NanoSEM 230) at $5 \mathrm{kV}$. The samples were coated with a thin layer of gold before measurement. The sessile drop method was used for water contact angle (WCA) measurements of the as-prepared surfaces using a data-physics OCA20 contact angle system at ambient temperature. The water droplet size used for measurements was $5.0 \mu \mathrm{L}$. The average WCA value was obtained by measuring more than five different positions for the same sample. The crystal structure of the samples was characterized by X-ray diffraction (XRD) on PANalytical X'Pert Pro. MPD X-ray diffractometer with $\mathrm{Ni}$ filtered $\mathrm{Cu} \mathrm{K} \alpha$ radiation of $1.542 \AA$ at the scan rate of $0.08^{\circ} \mathrm{s}^{-1}$. The chemical bonding mode of the films fabricated on aluminum substrate was investigated by Fourier transform infrared spectrometer (FTIR) spectroscopy (Shimadzu IRAffinity-1), and the films were scratched from aluminum substrate and pressed into tablets with $\mathrm{KBr}$. The chemical composition of the sample's surface was characterized using X-ray photoelectron spectra (XPS) in a Kratos Axis Ultra DLD system, using $\mathrm{Al} \mathrm{K \alpha}$ radiation as the exciting source. All binding energies in the spectra were corrected using standard binding energy of $\mathrm{C}$ 1s peak $(284.6 \mathrm{eV})$ as reference. Electrochemical impedance spectroscopy (EIS) was performed on a CHI 604C electrochemical analyzer (Shanghai ChenHua Instrument, China) in $3.5 \mathrm{wt} \% \mathrm{NaCl}$ aqueous solution at room temperature with a three-electrode system. An untreated or superhydrophobic aluminum plate was used as the working electrode, a platinum plate, and a saturated calomel electrode (SCE) were used as the counter and reference electrodes, respectively. The potentiodynamic polarization curves were subsequently measured with respect to the open circuit potential (OCP) at a scanning rate of $10 \mathrm{mV} / \mathrm{s}$ from -1100 to $+400 \mathrm{mV}$.

\section{Results and Discussion}

3.1. Morphology and Wettability of Sample. Figure 1 shows typical SEM images of (a) the untreated aluminum substrate and the sample surfaces after in situ deposition in zinc nitrate aqueous solution under different molar ratios between $\mathrm{Zn}\left(\mathrm{NO}_{3}\right)_{2} \cdot 6 \mathrm{H}_{2} \mathrm{O}$ and $\mathrm{CO}\left(\mathrm{NH}_{2}\right)_{2}:$ (b) $10: 1$; (c) $5: 1$; (d) $2: 1$; (e) $1: 2$; (f) $1: 5$. The wetting properties of the sample surfaces fabricated by in situ deposition in a zinc nitrate aqueous solution for $1.5 \mathrm{~h}$ followed by modification with FAS were evaluated by water contact angle measurements. It can be found from Figure 1(a) that the aluminum substrate was smooth and its water contact angle (WCA) was $88.1^{\circ}$, that is, the hydrophilic nature of aluminum substrate. There were different morphologies and WCAs after in situ immersion in the solution containing different molar ratios between $\mathrm{Zn}\left(\mathrm{NO}_{3}\right)_{2} \cdot 6 \mathrm{H}_{2} \mathrm{O}$ and $\mathrm{CO}\left(\mathrm{NH}_{2}\right)_{2}$. When the molar ratio between $\mathrm{Zn}\left(\mathrm{NO}_{3}\right)_{2} \cdot 6 \mathrm{H}_{2} \mathrm{O}$ and $\mathrm{CO}\left(\mathrm{NH}_{2}\right)_{2}$ was $10: 1$, the microstructure of the deposited film was microporous and the pore size ranged from $1 \mu \mathrm{m}$ to $3 \mu \mathrm{m}$, and the WCA was $128.7^{\circ}$ after modification, as shown in Figure 1(b). When the molar ratio was $5: 1$, the surface displayed rose petal-like microstructure composed of about $1 \mu \mathrm{m}$ sized petals, and WCA was $137.3^{\circ}$ after modification (Figure 1(c)). While when the molar ratio was $2: 1$, the surface displayed block-shaped microstructure with the size of each block ranging 200$300 \mathrm{~nm}$, and WCA was $156.2^{\circ}$ after modification (Figure 1(d)). 


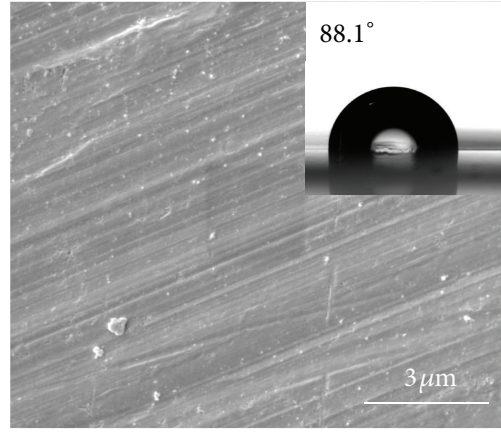

(a)

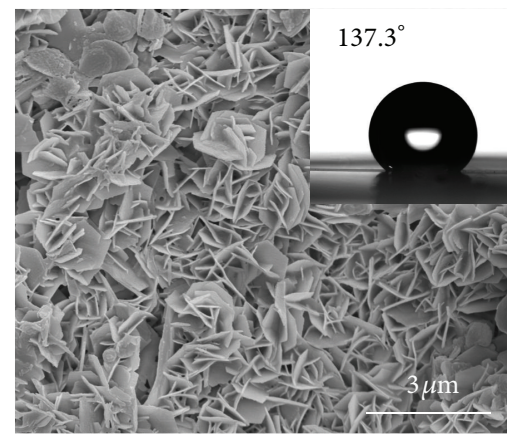

(c)

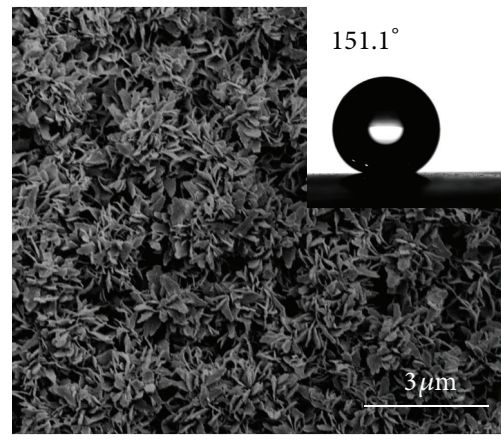

(e)

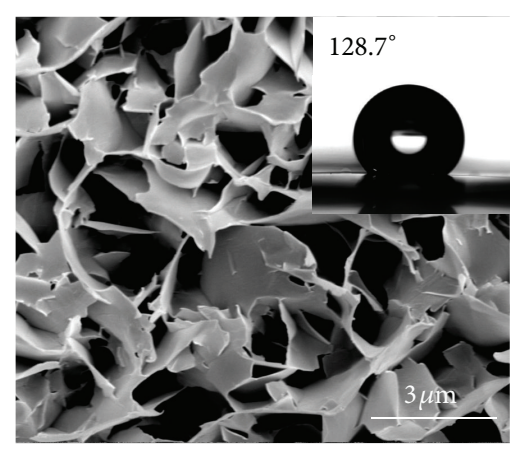

(b)

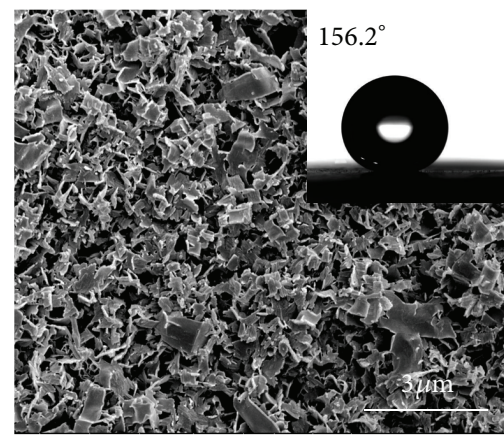

(d)

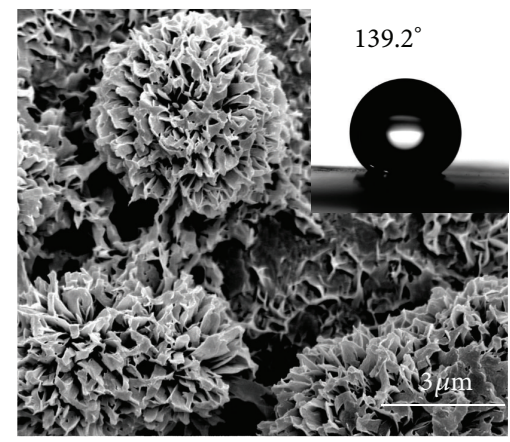

(f)

FIGURE 1: SEM images of (a) untreated aluminum and sample surfaces after immersion in zinc nitrate aqueous solution under the molar ratio between $\mathrm{Zn}\left(\mathrm{NO}_{3}\right)_{2} \cdot 6 \mathrm{H}_{2} \mathrm{O}$ and $\mathrm{CO}\left(\mathrm{NH}_{2}\right)_{2}$ which was (b) $10: 1$; (c) $5: 1 ;$ (d) $2: 1$; (e) $1: 2$; (f) $1: 5$.

When the molar ratios between $\mathrm{Zn}\left(\mathrm{NO}_{3}\right)_{2} \cdot 6 \mathrm{H}_{2} \mathrm{O}$ and $\mathrm{CO}\left(\mathrm{NH}_{2}\right)_{2}$ were $1: 2$ and $1: 5$, the surface displayed pineconelike microstructure with the size of each pinecone of around $2 \mu \mathrm{m}$ and $5 \mu \mathrm{m}$, respectively, and the corresponding WCA was $151.1^{\circ}$ and $139.2^{\circ}$ (Figures $1(\mathrm{e})$ and $1(\mathrm{f})$ ). Obviously, the molar ratio between $\mathrm{Zn}\left(\mathrm{NO}_{3}\right)_{2} \cdot 6 \mathrm{H}_{2} \mathrm{O}$ and $\mathrm{CO}\left(\mathrm{NH}_{2}\right)_{2}$ had a great influence on the morphology and wettability of the sample surfaces.

It is well known that low surface energy combined with surface roughness contribute to the superhydrophobic property of the surface [25]. Cassie and Baxter [26] proposed an equation to describe the relationship between the WCA on a flat surface $(\theta)$ and a rough heterogeneous surface $\left(\theta_{r}\right)$ composed of solid and air:

$$
\cos \theta_{r}=f_{1} \cos \theta-f_{2} .
$$

In this equation, $f_{1}$ and $f_{2}$ are the fraction of solid surface and trapped air in the composite surface, respectively (i.e., $f_{1}+f_{2}=1$ ). This equation predicts that the solid surface fraction $f_{1}$ is definitely important in determining the hydrophobicity of the heterogeneous surface; when the solid surface fraction $f_{1}$ is decreased, the WCA of rough surface can be increased, and at the limit when $f_{1}$ approaches zero, the surface contact angle approaches $180^{\circ}$. Substituting the WCA values on flat aluminum surface $\left(88.1^{\circ}\right)$ and superhydrophobic surface $\left(156.2^{\circ}\right)$ into (1), the value $f_{1}$ and $f_{2}$ of the heterogeneous aluminum surface can be calculated to be 0.082 and 0.918 , respectively. This means that air occupies about $91.8 \%$ of the contact areas; when the heterogeneous aluminum surface contacts with the water droplet, air pockets trap between the solid and liquid (the composite 


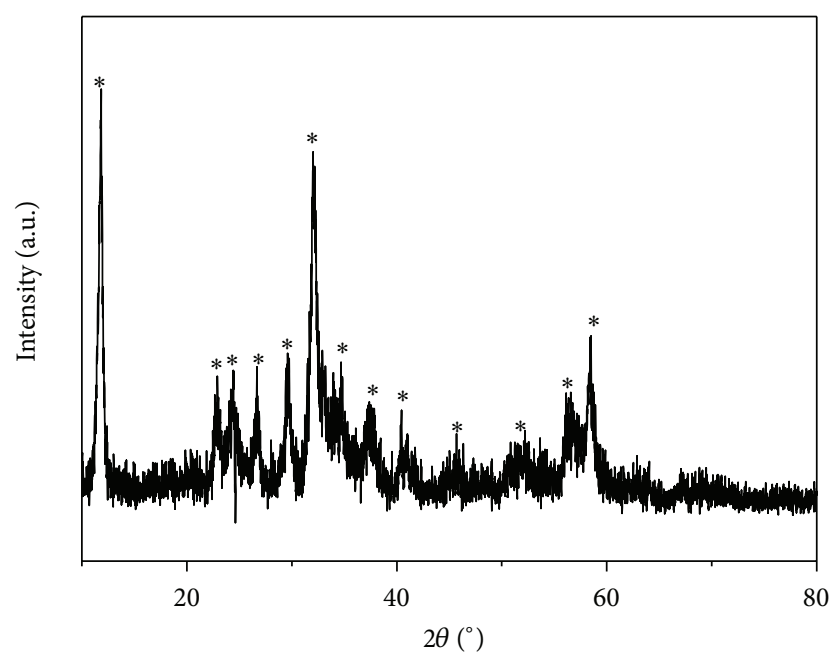

* $\mathrm{Zn}_{5}(\mathrm{OH})_{6}\left(\mathrm{CO}_{3}\right)_{2}$

FIGURE 2: XRD pattern of the powder obtained under the molar ratio of $2: 1$.

solid-liquid-air interface) and the droplet will be suspended upon the asperity, thus leading to a significant increase of WCA [27]. In summary, the molar ratio of $2: 1$ between $\mathrm{Zn}\left(\mathrm{NO}_{3}\right)_{2} \cdot 6 \mathrm{H}_{2} \mathrm{O}$ and $\mathrm{CO}\left(\mathrm{NH}_{2}\right)_{2}$ was the best condition to create appropriate microstructure and roughness on the surface, and thus lay the morphological foundation for the excellent superhydrophobicity.

3.2. Sample Structure and Chemical Composition of the Superhydrophobic Surface. XRD was employed to analyze the chemical structure of the sample. The XRD pattern of powder deposited under the molar ratio of $2: 1$ was shown in Figure 2. All of the reflection peaks can be indexed to hydroxide zinc carbonate $(\mathrm{HZC}), \mathrm{Zn}_{5}(\mathrm{OH})_{6}\left(\mathrm{CO}_{3}\right)_{2}$ (in Joint Committee on Powder Diffraction Standards, JCPDS card no. 19-1458). The formation of HZC crystals was initiated by the slow reaction between urea and water. With the hydrolysis of urea, carbonate and hydroxyl being slowly released, $\mathrm{Zn}$ ions in the aqueous solution undergo a homogeneous coprecipitation with released carbonate and hydroxyl and finally lead to the formation of HZC $[28,29]$.

The chemical composition and bonding states of the superhydrophobic films were investigated by FTIR and XPS analysis. The FTIR spectrum of Figure 1(d) sample was shown in Figure 3. A broad absorption band coming from $-\mathrm{OH}$ groups appears at around $3421 \mathrm{~cm}^{-1}$. The absorption bands corresponding to the $\mathrm{CO}_{3}{ }^{2-}$ group can also be observed: the absorption bands at $1552 \mathrm{~cm}^{-1}$ and $1382 \mathrm{~cm}^{-1}$ can be assigned to the asymmetric stretching modes and the band at $829 \mathrm{~cm}^{-1}$ should be attributed to the out-of-plane deformation mode [24]. The presence of the " $\mathrm{Zn}-\mathrm{OH}$ " bonding can also be observed at $941 \mathrm{~cm}^{-1}$ [30]. The bands at 2926 and $2854 \mathrm{~cm}^{-1}$ should be attributed to asymmetric and symmetric stretching vibrations of $\mathrm{C}-\mathrm{H}$ groups, respectively [31], while the band at $1041 \mathrm{~cm}^{-1}$ can be assigned to $\mathrm{C}-\mathrm{F}$ stretching of $-\mathrm{CF}_{2}$ group

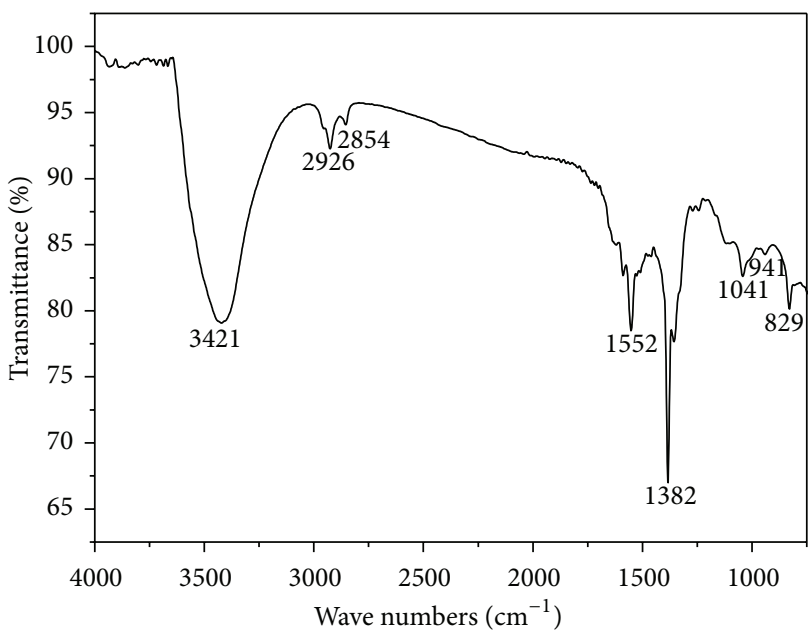

FIGURE 3: FTIR spectrum of the superhydrophobic film.

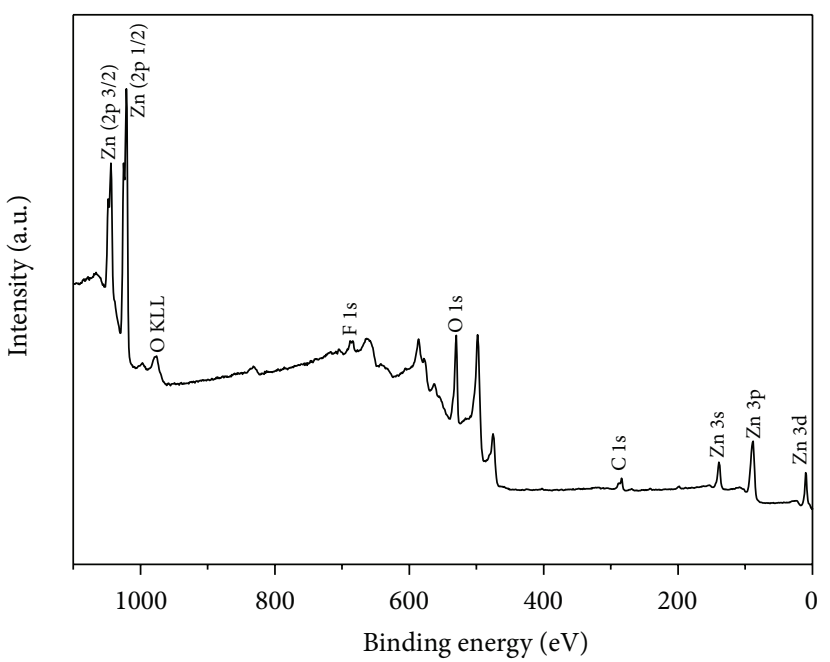

FIGURE 4: XPS spectra of the superhydrophobic film on aluminum substrate.

[32], which, implying the long-chain alkyl group from FAS, is chemically absorbed on the surface.

The XPS spectrum of the superhydrophobic surface was shown in Figure 4. Peaks due to C 1s $(284 \mathrm{eV}), \mathrm{O}$ 1s $(530 \mathrm{eV})$, and $\mathrm{F}$ 1s $(686 \mathrm{eV})$ were observed. The presence of the $\mathrm{F}$ 1s peak and $\mathrm{C}$ 1s peak (due to $\mathrm{C}-\mathrm{F}$ ) confirms that FAS molecules has been successfully self-assembled onto the HZC surface [33]. This is in good accordance with the results of FTIR. The analysis of FTIR and XPS reflects that the HZC film was covered with fluorine containing long-chain alkyl chemical species, inducing the low surface energy surface, which combined with rough microstructure of $\mathrm{HZC}$ film, to obtain excellent superhydrophobicity of the sample.

3.3. Electrochemical Impedance Analysis. Figure 5 shows the potentiodynamic polarization curves of (a) untreated aluminum and (b) superhydrophobic aluminum. As can be clearly seen in Figure 5, the corrosion potential, $E_{\text {corr }}$, of the superhydrophobic surface was positive than that of the 


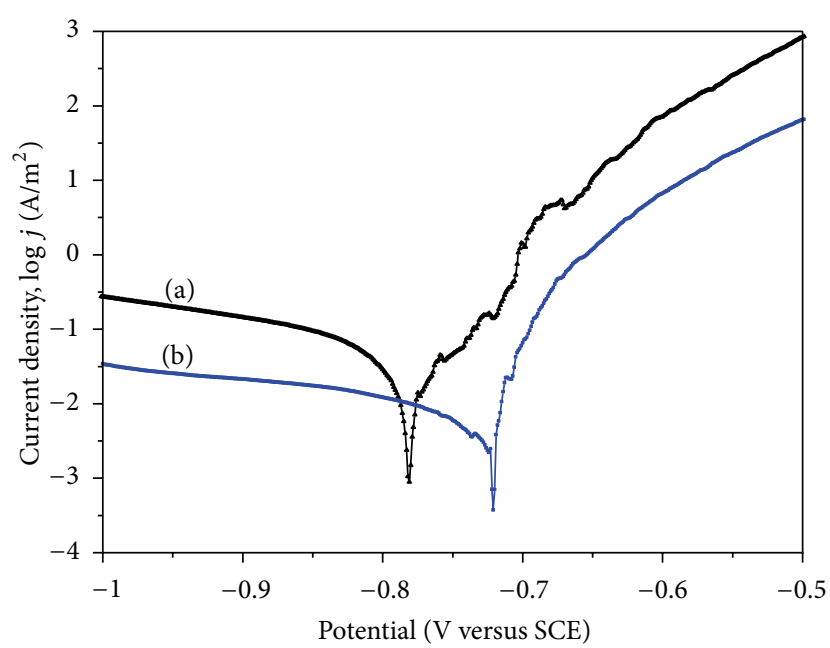

(a) Untreated aluminum

(b) Superhydrophobic surface

FIGURE 5: Potentiodynamic polarization curves of (a) untreated aluminum and (b) superhydrophobic aluminum in $3.5 \mathrm{wt} \% \mathrm{NaCl}$ solution.

untreated aluminum. The shift of the $E_{\text {corr }}$ towards the positive direction could be linked to an improvement of the protective properties of the superhydrophobic film formed on aluminum substrate. The corrosion current density, $j_{\text {corr }}$, of the superhydrophobic surface on aluminum substrate $(7.53 \times$ $10^{-3} \mathrm{~A} / \mathrm{m}^{2}$ ) decreased by more than 1 order of magnitude as compared to that of the untreated one $\left(2.92 \times 10^{-2} \mathrm{~A} / \mathrm{m}^{2}\right)$. These results indicate that the superhydrophobic film has good corrosion resistance.

\section{Conclusions}

In conclusion, superhydrophobic surfaces were successfully fabricated on aluminum by a two-step method. In the initial step, HZC films with different microstructures were fabricated on aluminum via a convenient in situ immersion in zinc nitrate aqueous solution by adjusting the molar ratios between $\mathrm{Zn}\left(\mathrm{NO}_{3}\right)_{2} \cdot 6 \mathrm{H}_{2} \mathrm{O}$ and $\mathrm{CO}\left(\mathrm{NH}_{2}\right)_{2}$. In the second step, the HZC surfaces were modified with FAS molecules. All FAS-modified HZC surfaces enhanced the water repellent property of the aluminum and obtained a greatest static contact angle of $156.2^{\circ}$, indicating excellent superhydrophobicity. Moreover, it is significant that the electrochemical measurements' results revealed that the superhydrophobic aluminum has good corrosion resistance in corrosive medium.

\section{Acknowledgments}

This work was financially supported by the National Natural Science Foundation of China (Grant no. 31270602 and 31270603), the Science and Technology Projects Foundation of Hunan Province (Grant no. 2012GK3020), and Youth Scientific Research Foundation of Central South University of Forestry and Technology (Grant no. QJ2011021B).

\section{References}

[1] A. M. Abdel-Gaber, B. A. Abd-El-Nabey, I. M. Sidahmed, A. M. El-Zayady, and M. Saadawy, "Kinetics and thermodynamics of aluminium dissolution in $1.0 \mathrm{M}$ sulphuric acid containing chloride ions," Materials Chemistry and Physics, vol. 98, no. 2-3, pp. 291-297, 2006.

[2] B. Yin, L. Fang, A. Q. Tang et al., "Novel strategy in increasing stability and corrosion resistance for super-hydrophobic coating on aluminum alloy surfaces," Applied Surface Science, vol. 258, no. 1, pp. 580-585, 2011.

[3] W. A. Badawy, F. M. Al-Kharafi, and A. S. El-Azab, "Electrochemical behaviour and corrosion inhibition of Al, Al-6061 and $\mathrm{Al}-\mathrm{Cu}$ in neutral aqueous solutions," Corrosion Science, vol. 41, no. 4, pp. 709-727, 1999.

[4] X. Feng and L. Jiang, "Design and creation of superwetting/antiwetting surfaces," Advanced Materials, vol. 18, no. 23, pp. 30633078, 2006.

[5] X. M. Li, D. Reinhoudt, and M. Crego-Calama, "What do we need for a superhydrophobic surface? A review on the recent progress in the preparation of superhydrophobic surfaces," Chemical Society Reviews, vol. 36, no. 8, pp. 1350-1368, 2007.

[6] P. Roach, N. J. Shirtcliffe, and M. I. Newton, "Progess in superhydrophobic surface development," Soft Matter, vol. 4, no. 2, pp. 224-240, 2008.

[7] W. Barthlott and C. Neinhuis, "Purity of the sacred lotus, or escape from contamination in biological surfaces," Planta, vol. 202, no. 1, pp. 1-8, 1997.

[8] E. Burkarter, C. K. Saul, F. Thomazi et al., "Electrosprayed superhydrophobic PTFE: a non-contaminating surface," Journal of Physics D, vol. 40, no. 24, pp. 7778-7781, 2007.

[9] B. Qian and Z. Shen, "Fabrication of superhydrophobic surfaces by dislocation-selective chemical etching on aluminum, copper, and zinc substrates," Langmuir, vol. 21, no. 20, pp. 9007-9009, 2005.

[10] L. Y. Meng and S. J. Park, "Effect of fluorination of carbon nanotubes on superhydrophobic properties of fluoro-based films," Journal of Colloid and Interface Science, vol. 342, no. 2, pp. 559$563,2010$.

[11] W. Y. Gan, S. W. Lam, K. Chiang, R. Amal, H. Zhao, and M. P. Brungs, "Novel $\mathrm{TiO}_{2}$ thin film with non-UV activated superwetting and antifogging behaviours," Journal of Materials Chemistry, vol. 17, no. 10, pp. 952-954, 2007.

[12] M. Li, J. Zhai, H. Liu, Y. Song, L. Jiang, and D. Zhu, "Electrochemical deposition of conductive superhydrophobic zinc oxide thin films," Journal of Physical Chemistry B, vol. 107, no. 37, pp. 9954-9957, 2003.

[13] L. Zhang, Z. Zhou, B. Cheng, J. M. DeSimone, and E. T. Samulski, "Superhydrophobic behavior of a perfluoropolyether lotusleaf-like topography," Langmuir, vol. 22, no. 20, pp. 8576-8580, 2006.

[14] A. Nakajima, K. Abe, K. Hashimoto, and T. Watanabe, "Preparation of hard super-hydrophobic films with visible light transmission," Thin Solid Films, vol. 376, no. 1-2, pp. 140-143, 2000.

[15] A. Venkateswara Rao, S. S. Latthe, D. Y. Nadargi, H. Hirashima, and V. Ganesan, "Preparation of MTMS based transparent superhydrophobic silica films by sol-gel method," Journal of Colloid and Interface Science, vol. 332, no. 2, pp. 484-490, 2009.

[16] N. Zhao, F. Shi, Z. Wang, and X. Zhang, "Combining layer-bylayer assembly with electrodeposition of silver aggregates for fabricating superhydrophobic surfaces," Langmuir, vol. 21, no. 10, pp. 4713-4716, 2005. 
[17] H. J. Tsai and Y. L. Lee, "Facile method to fabricate raspberrylike particulate films for superhydrophobic surfaces," Langmuir, vol. 23, no. 25, pp. 12687-12692, 2007.

[18] L. Liu, J. Zhao, Y. Zhang, F. Zhao, and Y. Zhang, "Fabrication of superhydrophobic surface by hierarchical growth of lotus-leaflike boehmite on aluminum foil," Journal of Colloid and Interface Science, vol. 358, no. 1, pp. 277-283, 2011.

[19] W. Wu, M. Chen, S. Liang, X. Wang, J. Chen, and F. Zhou, "Superhydrophobic surface from $\mathrm{Cu}-\mathrm{Zn}$ alloy by one step $\mathrm{O}_{2}$ concentration dependent etching," Journal of Colloid and Interface Science, vol. 326, no. 2, pp. 478-482, 2008.

[20] T. Ishizaki and N. Saito, "Rapid formation of a superhydrophobic surface on a magnesium alloy coated with a cerium oxide film by a simple immersion process at room temperature and its chemical stability," Langmuir, vol. 26, no. 12, pp. 9749-9755, 2010.

[21] T. Ishizaki, Y. Masuda, and M. Sakamoto, "Corrosion resistance and durability of superhydrophobic surface formed on magnesium alloy coated with nanostructured cerium oxide film and fluoroalkylsilane molecules in corrosive $\mathrm{NaCl}$ aqueous solution," Langmuir, vol. 27, no. 8, pp. 4780-4788, 2011.

[22] Y. W. Hu, S. Y. Huang, S. Liu et al., "A corrosion-resistance superhydrophobic $\mathrm{TiO}_{2}$ film," Applied Surface Science, vol. 258, no. 19, pp. 7460-7464, 2012.

[23] F. Zhang, S. Chen, L. Dong, Y. Lei, T. Liu, and Y. Yin, "Preparation of superhydrophobic films on titanium as effective corrosion barriers," Applied Surface Science, vol. 257, no. 7, pp. 2587-2591, 2011.

[24] B. Su, M. Li, Z. Shi, and Q. Lu, "From superhydrophilic to superhydrophobic: controlling wettability of hydroxide zinc carbonate film on zinc plates," Langmuir, vol. 25, no. 6, pp. 3640-3645, 2009.

[25] M. Nosonovsky and B. Bhushan, "Superhydrophobic surfaces and emerging applications: non-adhesion, energy, green engineering," Current Opinion in Colloid and Interface Science, vol. 14, no. 4, pp. 270-280, 2009.

[26] A. B. D. Cassie and S. Baxter, "Wettability of porous surfaces," Transactions of the Faraday Society, vol. 40, pp. 546-551, 1944.

[27] Z. Yoshimitsu, A. Nakajima, T. Watanabe, and K. Hashimoto, "Effects of surface structure on the hydrophobicity and sliding behavior of water droplets," Langmuir, vol. 18, no. 15, pp. 5818$5822,2002$.

[28] L. Xu, Y. S. Ding, C. H. Chen et al., "3D flowerlike $\alpha$-nickel hydroxide with enhanced electrochemical activity synthesized by microwave-assisted hydrothermal method," Chemistry of Materials, vol. 20, no. 1, pp. 308-316, 2008.

[29] X. F. Zhou, Z. L. Hu, Y. Q. Fan et al., "Microspheric organization of multilayered $\mathrm{ZnO}$ nanosheets with hierarchically porous structures," Journal of Physical Chemistry C, vol. 112, no. 31, pp. 11722-11728, 2008.

[30] K. Kakiuchi, E. Hosono, T. Kimura, H. Imai, and S. Fujihara, "Fabrication of mesoporous $\mathrm{ZnO}$ nanosheets from precursor templates grown in aqueous solutions," Journal of Sol-Gel Science and Technology, vol. 39, no. 1, pp. 63-72, 2006.

[31] Y. Wang, W. Wang, L. Zhong, J. Wang, Q. Jiang, and X. Guo, "Super-hydrophobic surface on pure magnesium substrate by wet chemical method," Applied Surface Science, vol. 256, no. 12, pp. 3837-3840, 2010.

[32] H. J. Jeong, D. K. Kim, S. B. Lee, S. H. Kwon, and K. Kadono, "Preparation of water-repellent glass by sol-gel process using perfluoroalkylsilane and tetraethoxysilane," Journal of Colloid and Interface Science, vol. 235, no. 1, pp. 130-134, 2001.
[33] A. Delimi, E. Galopin, Y. Coffinier et al., "Investigation of the corrosion behavior of carbon steel coated with fluoropolymer thin films," Surface and Coatings Technology, vol. 205, no. 16, pp. 4011-4017, 2011. 

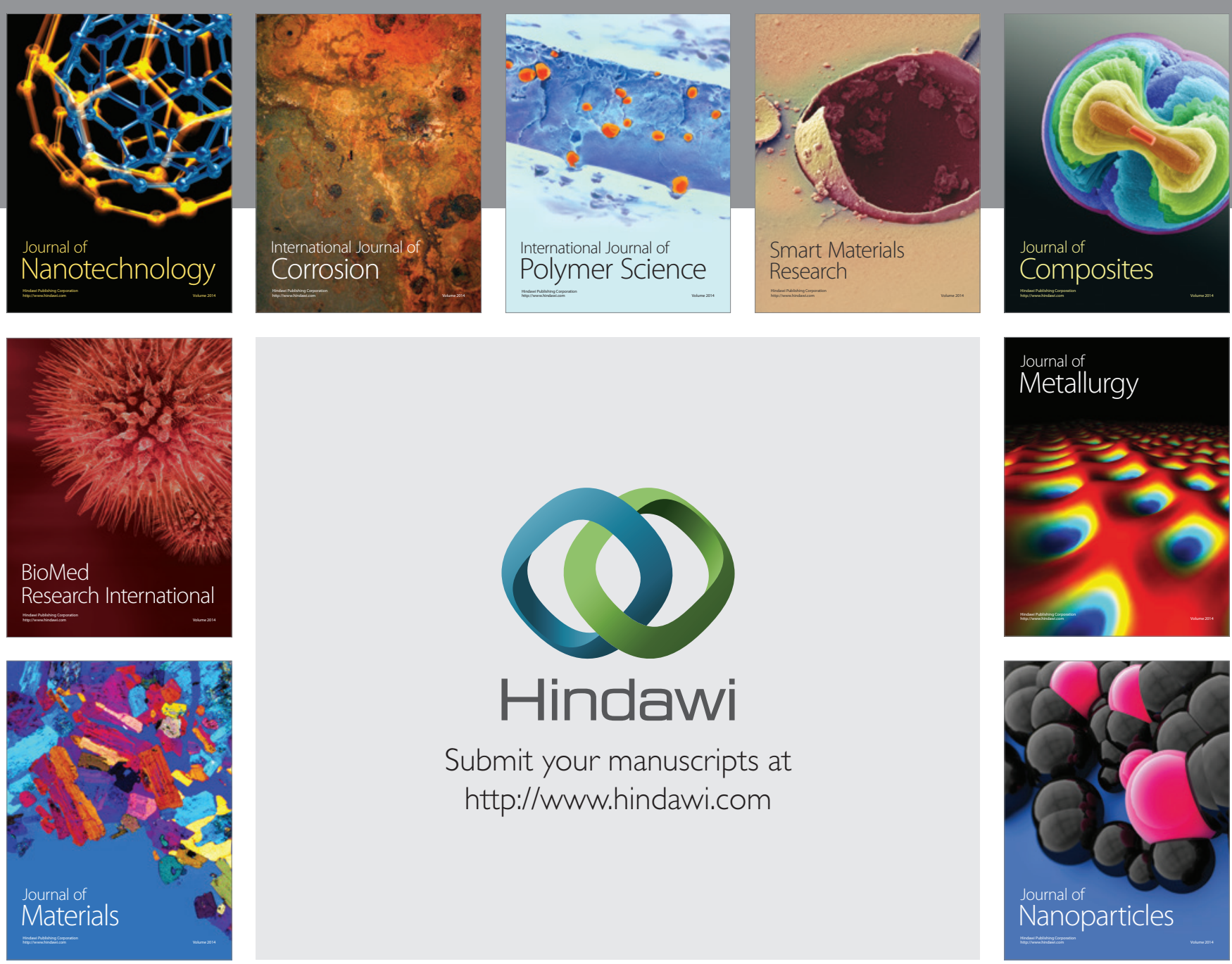

Submit your manuscripts at http://www.hindawi.com
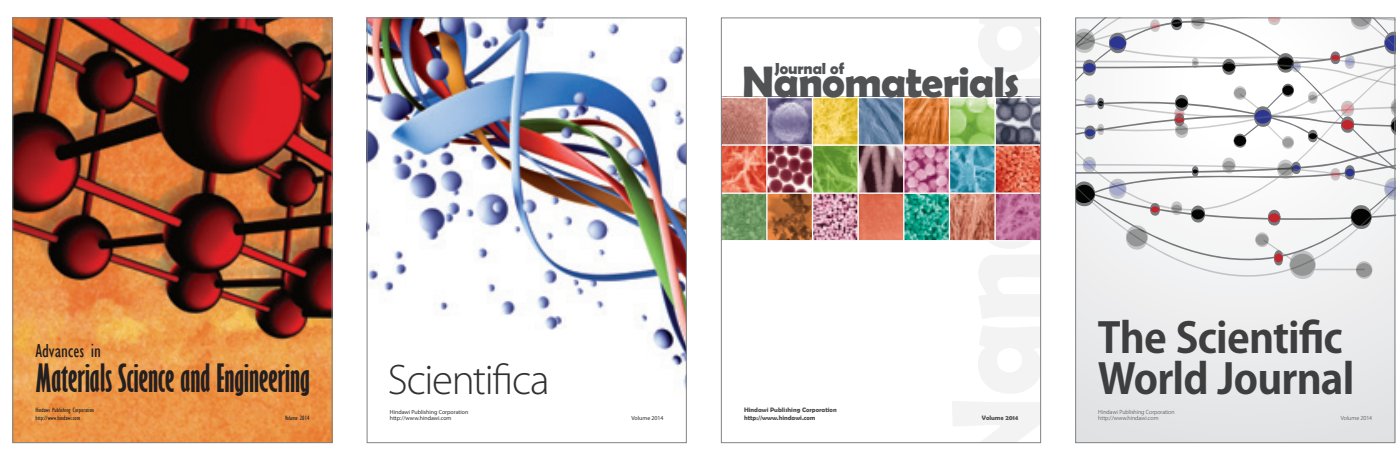

\section{The Scientific World Journal}
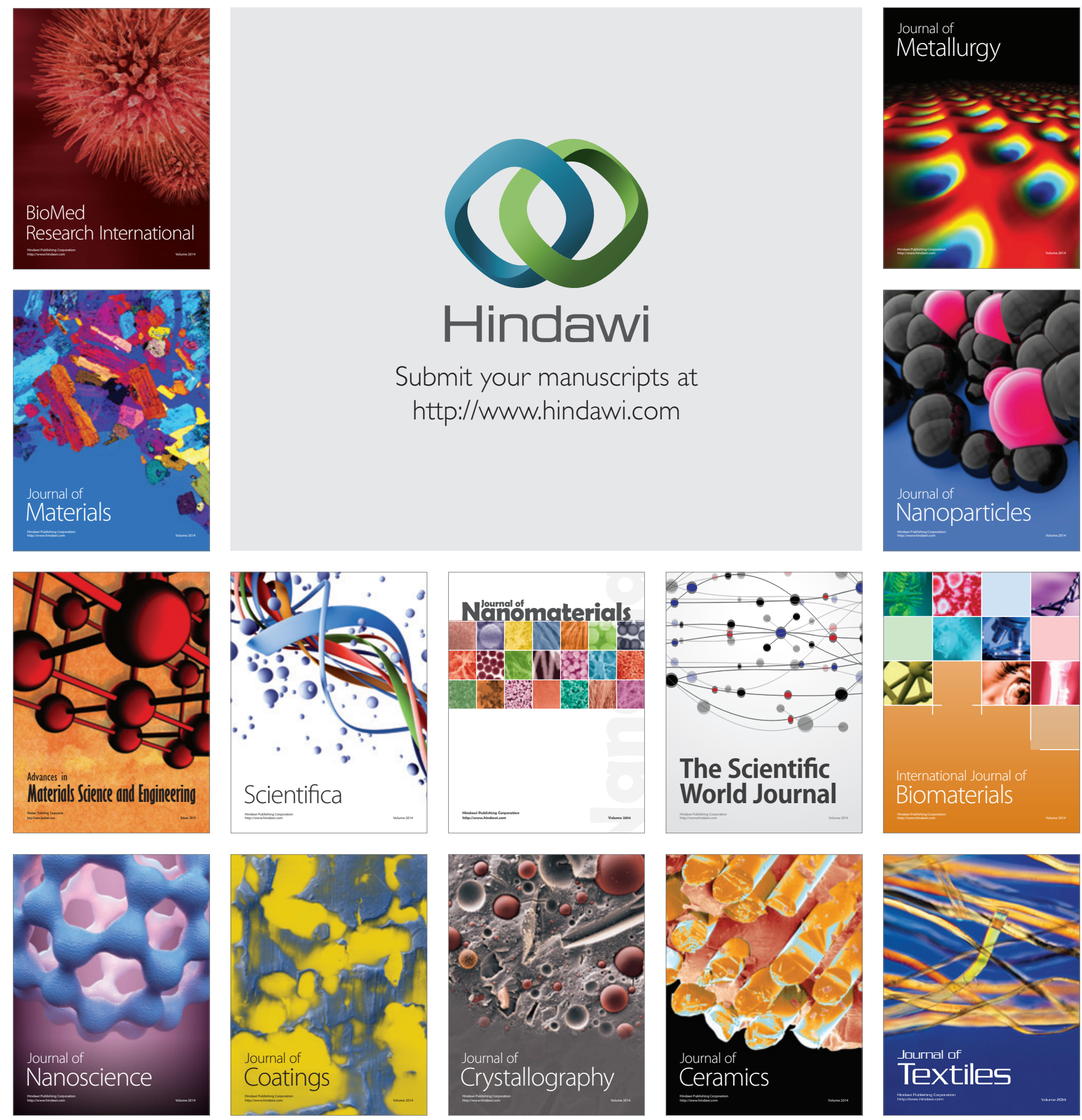